\title{
Effects of massage therapy and aromatherapy on fatigue in patients with knee osteoarthritis
}

\author{
Fariba Kabiri ${ }^{1}$, Ali Hasanpour-Dehkordi ${ }^{*}$, Fatemeh Dris ${ }^{3}$ \\ ${ }^{1}$ Treatment Management Organization in Chaharmahal and Bakhtiari province, Social Security Hospital of Imam Ali (AS), Shahrekord, Iran \\ ${ }^{2}$ School of Allied Medical Sciences, Shahrekord University of Medical Sciences, Shahrekord, Iran \\ ${ }^{3}$ Medical Plants Research Center, Basic Health Sciences Institute, Shahrekord University of Medical Sciences, Shahrekord, Iran
}

\section{A R T I C L E I N F O}

Article Type:

Original Article

\section{Article History:}

Received: 10 January 2018

Accepted: 5 June 2018

\section{Keywords:}

Aromatherapy

Lavender

Massage

Knee osteoarthritis

Fatigue

\begin{abstract}
A B S T R A C T
Introduction: Knee osteoarthritis is the most common articular cartilage disease. The purpose of this study was to compare the effects of massage therapy and aromatherapy (Lavender essential oil) on fatigue in patients with knee osteoarthritis.

Methods: In this clinical trial, 93 patients referred to the Imam Ali Clinic in Shahrekord, Iran, were included. The patients were randomly assigned to 3 equal groups of massage therapy, aromatherapy (Lavender essential oil), and control by blocked randomization. Data were collected using demographic characteristics questionnaire and the Multidimensional Fatigue Inventory (MFI). Fatigue scores in the three groups were determined before intervention and in the 5th and 10th sessions after intervention.

Results: No significant differences in demographic characteristics were observed between the groups. There was a significant reduction in fatigue score in the aromatherapy group when compared to the control group $(P<0.001)$. However, no statistically significant difference was observed in fatigue scores at different intervals between the control and massage therapy groups.

Conclusion: Since knee osteoarthritis has no definite treatment, aromatherapy can lead to improving fatigue in patients with the disease.
\end{abstract}

Implication for health policy/practice/research/medical education:

Aromatherapy (Lavender essential oil) significantly reduced fatigue in patients with knee osteoarthritis. Thus, due to the high prevalence of knee osteoarthritis, this method is recommended to patents with osteoarthritis because of its availability, security, and cost-effectiveness.

Please cite this paper as: Kabiri F, Hasanpour-Dehkordi A, Dris F. Effects of massage therapy and aromatherapy on fatigue in patients with knee osteoarthritis. J Herbmed Pharmacol. 2018;7(3):141-147. doi: 10.15171/jhp.2018.24.

\section{Introduction}

Knee osteoarthritis is a multifactorial degenerative joint disease that is caused by changes in the articular cartilage and the sub-chondrial bone, osteophyte formation, and synovial tissue inflammation (1). Osteoarthritis is one of the most common articular cartilage diseases and one of the major causes of physical and socioeconomic disabilities (2). The disease comprises half of the problems in people aged over 65 years and represents the second leading complaint among outpatients. Osteoarthritis brings discomfort and disability for around $10 \%$ of the total human population due to chronic joint pain and inflammation. Although knee osteoarthritis is not a fatal disease, if left untreated, most of the patients will have to tolerate chronic joint pain and join diseases until the end of their life (3).

An estimate of over 50 million Americans are expected to develop osteoarthritis by 2020 (4).

In Iran, nine out of every 10 patients aged $30-35$ years who refer with the main complaints related to knee, waist, and neck pain suffer from osteoarthritis. Patients with osteoarthritis are reported to visit the doctor approximately 8 times each year, which is twice as high as the number of referrals by patients with hypertension (5). The main symptoms of knee osteoarthritis include pain, morning stiffness, mobility impairments, and articular tenderness (6). Fatigue is a multidimensional concept that includes general fatigue, physical fatigue, mental fatigue, reduced activity, and reduced motivation. General fatigue affects the individual's general functions during 
the day, physical fatigue refers to the fatigue-related bodily sensations, mental fatigue is characterized by the development of cognitive symptoms, reduced activity is related to decline in activity after development of mental fatigue, and reduced motivation refers to a reduction or lack of motivation to start any activity (7).

Conventional treatments for osteoarthritis include nonsteroidal anti-inflammatory drugs (NSAIDs), cyclooxygenases, corticosteroids, intra-articular injection of hyaluronic acid, and ultimately joint replacement. Most of these drugs produce numerous toxic effects $(8,9)$. Therefore, the use of nonpharmacologic methods has been given priority due to their ease of use, increase in the activity and compliance of the patients, reduction in health care costs, hospital bed occupancy rate, and costeffectiveness (10).

Aromatherapy, massage therapy, muscle relaxation, music therapy, guided imagery, yoga, and hypnosis have been considered alternative or complementary approaches (1113) Massage is defined as a manual work on soft tissues of the body with a rhythmic and impulsive pressure aimed at improving the health and well-being of the body and includes a variety of manual techniques, pressure techniques, and rubbing the body. Since massage reduces the activity of the adrenal gland, level of adrenalin, activity of the sympathetic nervous system, and stress, it indirectly affects pain relief (14).

Besides that, aromatherapy is used to relieve pain, anxiety, depression, insomnia, and fatigue by means of the essential oils extracted from different parts of the plants. Aromatherapy gently induces certain changes in the mind and body, and reduces muscle pain (15). Lavender is one of the most widely used medicinal plants in aromatherapy. It is one of the most popular essential oils for relieving pain. It is a safe plant without any known contraindications, and has been frequently used in different studies $(16,17)$.

Maintenance of physical function to do self-care activities is essential for patients with chronic diseases such as osteoarthritis; and complementary methods can reduce the severity of pain and symptoms, and improve motor function in such patients. More importantly, in Iran, due to cultural differences in people's lifestyle and customs, including the habit of sitting on the ground, the use of traditional toilets, and low physical activity, and the debilitating nature of osteoarthritis, the disease is more prevalent. Therefore, the aim of this study was to compare the effects of massage therapy and aromatherapy on fatigue in patients with knee osteoarthritis referred to the Imam Ali Clinic in Shahrekord, Iran.

\section{Materials and Methods}

The inclusion criteria consisted of patients suffering from knee osteoarthritis according to the diagnosis of rheumatologist, having suffered from the disease for at least six months and at most five years, lack of topical skin infections and open wounds in the areas where massage would be done, being 40-60 years old, lack of history of other autoimmune diseases such as lupus erythematous and rheumatoid arthritis, lack of history of chronic neuropathic diseases such as diabetes, lack of intraarticular drug injection in the past three months, lack of previous knee surgery and severe knee trauma, lack of history of asthma, migraine, allergy to plants, chronic headaches, and heart disease, and having a healthy sense of smell. Exclusion criteria were exacerbation of the disease symptoms, lack of regular referring to the clinic, and the use of NSAIDs for knee pain during the study. After obtaining written consent from the patients, participants were selected by convenience sampling and assigned to three equal groups by blocked randomization.

The instrument for collecting the data included a questionnaire, including demographic characteristics (gender, age, height, and weight), number of children, occupation, physicalactivity, and the duration of the disease, as well as four items about the history of allergy to plants, migraines, chronic headaches, and olfactory impairment. This questionnaire was filled out by the researcher as he was interviewing the participants. The second section of the instrument used was the Multidimensional Fatigue Inventory (MFI). This inventory has 20 items and covers five dimensions: General fatigue (items 1, 5, 12, and 16), physical fatigue (items $2,8,14$, and 20), mental fatigue (items $7,11,13$, and 19), reduced activity (items 3, 6, 10, and 17), and reduced motivation (items 4, 9, 15, and 18). The items are rated on a 5-point Likert scale from Yes, absolutely correct to No, absolutely incorrect. The score of each item ranges between 1 and 5, and the items 2, 5, 9, 13, $14,16,17,18$, and 19 are scored inversely. The total fatigue score is determined by summing scores on all dimensions ranges between 20 and 100, with higher scores indicating higher levels of fatigue. Validity and reliability of this instrument have been determined previously $(18,19)$. The MFI has also been translated into Persian and the Persian duplicate has been validated (20). It has also been used for various types of diseases such as chronic fatigue syndrome (21), cancer (22), heart failure, and respiratory disorders $(23,24)$ as well as in hemodialysis patients $(7)$.

All 3 groups underwent physiotherapy using TENS and FARADIC (Iran) at $50 \mathrm{~Hz}$ current.

After physiotherapy, classical massage was done on the knee by the researcher for the patients in the massage therapy group. It should be noted that the researcher himself completed the questionnaire and was present during the interventions.

Because the intervention was performed by manual massage, the researcher used sweet almond oil to reduce friction and slipperiness of the area on which massage would be done. The amount of oil used in each session was $3 \mathrm{~mL}$ and massage therapy was conducted within 10 sessions, each session lasting 20 minutes, and three sessions 
per week. For the aromatherapy group, the essential oil of lavender (botanically called Lavandula angustifolia) (Barij Essence Co., Kashan, Iran), which is a flowering plant of the Lavandula genus, was used. The patients in this group were asked to refer for physiotherapy in the morning. Then, two drops of L. angustifolia essential oil embedded in a $3 \mathrm{ml}$ dropper was dropped on a cotton ball and then the cotton ball was attached to the collar from 10:00 PM to 7:00 AM at home every other day for one month.

The control group received routine physiotherapy alone. Patients were asked to inform the researcher if they used the analgesics or if their symptoms were exacerbated. Such patients were excluded from the study.

The MFI was administered before intervention, and also in the fifth and tenth sessions of the intervention in all three groups. The patients in the aromatherapy group were asked to refer to the clinic after the tenth session of intervention to be followed up for adherence to the determined protocol.

Data analysis was conducted by the SPSS version 20 using chi-square, Fisher's exact test, repeated measures ANOVA, one-way ANOVA, and Tukey's test.

\section{Results}

In this study, 93 patients with knee osteoarthritis were studied in three groups (31 patients in each group), controls, massage therapy group, and aromatherapy group. The majority of participants were female and housewives. The mean ( \pm standard deviation) age of the participants was $72.5( \pm 9.5)$ years in the control group, $72.9( \pm 9.9)$ years in the massage therapy group, and $69.5( \pm 9.7)$ years in the aromatherapy group $(P>0.05)$.

The age, sex, body mass index (BMI), and other demographic variables of the three groups were not significantly different $(P>0.05)$ (Tables 1 and 2$)$. The repeated measures ANOVA also indicated no difference in fatigue score among the groups $(P=0.120)$. But, the mean scores at different intervals were significantly different $(P=0.003)$. The fatigue scores at different intervals were significantly different before intervention when compared to those after intervention, while no difference in pain score between the fifth and tenth sessions was observed. The mean scores of fatigue were significantly different between the groups $(P<0.001)$. At all intervals, the fatigue scores of the control group were higher than those of the other two groups and the fatigue score in the massage therapy group in the fifth session of intervention decreased when compared to that before the intervention, and in the tenth session, the fatigue score increased when compared to that in the fifth session. The fatigue score decreased at all intervals in the aromatherapy group (Table 3 ). After the completion of the intervention, the mean scores on the dimensions of the MFI were calculated separately, with the results as follows: Repeated measures ANOVA results on the general fatigue score showed that no statistically significant difference in the mean score of general fatigue was observed among the three groups $(P=0.772)$. The mean scores of general fatigue at different intervals were not statistically different $(P=0.058)$. The mean scores of general fatigue at different intervals in different groups were not significantly different $(P<0.001)$. At all intervals, the control group attained the highest general fatigue score, but the score decreased in both the massage therapy group and the aromatherapy group, with a more pronounced decrease in the massage therapy group (Table 4). Repeated measures ANOVA results on physical fatigue score showed that there was no significant difference in the mean scores of physical fatigue among the groups $(P=$ 0.108 ). The mean scores of physical fatigue at the studied intervals were not significantly different $(P=0.149)$. The mean scores of physical fatigue at different intervals were not significantly different among the three groups $(P=$

Table 1. Mean and standard deviation of statistical indicators distribution of demographic variables in the studied groups

\begin{tabular}{|c|c|c|c|c|c|}
\hline Variable & & Aromatherapy & Control & Massage therapy & $P$ value \\
\hline \multirow{2}{*}{ Sex } & Male & $10(32.3)$ & $10(32.3)$ & $10(32.3)$ & \multirow{2}{*}{1.000} \\
\hline & Female & $21(67.7)$ & $21(67.7)$ & $21(67.7)$ & \\
\hline \multirow{4}{*}{ Occupation } & Full time & $5(16.1)$ & $8(25.8)$ & $6(19.4)$ & \multirow{4}{*}{0.373} \\
\hline & Part time & $2(6.5)$ & $6(19.4)$ & 4 (12.9) & \\
\hline & Housewife & $14(45.2)$ & $12(38.7)$ & $17(54.8)$ & \\
\hline & Retired & $10(32.3)$ & $5(16.1)$ & $4(12.9)$ & \\
\hline
\end{tabular}

Table 2. Mean and standard deviation of statistical distribution of quantitative demographic variables in the studied groups

\begin{tabular}{lccc}
\hline Variable & Aromatherapy & Control & Massage therapy \\
\hline Age, year & $49.2 \pm 6.4$ & $50.2 \pm 6.2$ & $50.5 \pm 5.9$ \\
Weight, kg & $73.06 \pm 8.52$ & $73.90 \pm 11.37$ & $74.84 \pm 8.78$ \\
Height, cm & $164.87 \pm 6.20$ & $164.42 \pm 7.53$ & $164.48 \pm 6.51$ \\
BMI & $26.15 \pm 3.02$ & $25.96 \pm 3.82$ & $24.84 \pm 2.62$ \\
Number of children & $4.3 \pm 2.4$ & $3.4 \pm 1.7$ & $4.0 \pm 2.9$ \\
Activity, min & $3.53 \pm 2.19$ & $3.94 \pm 2.98$ & $3.55 \pm 2.79$ \\
Duration of disease, month & $23.16 \pm 19.15$ & $23.29 \pm 19.40$ & 0.960 \\
\hline
\end{tabular}


Table 3. Mean and standard deviation of fatigue score in the studied groups at the time of study

\begin{tabular}{lcccc}
\hline Time & Aromatherapy & Massage therapy & Control & Total \\
\hline Before intervention & $59.32 \pm 14.30$ & $59.13 \pm 8.18$ & $62.77 \pm 10.95$ & $60.41 \pm 10.95$ \\
After 5th session & $59.06 \pm 14.27$ & $57.23 \pm 7.46$ & $62.87 \pm 9.76$ & $59.71 \pm 11.01$ \\
After 10th session & $58.97 \pm 13.82$ & $59.10 \pm 7.02$ & $63.19 \pm 8.86$ & $59.42 \pm 10.61$ \\
Total & $59.12 \pm 1.90$ & $57.48 \pm 1.90$ & $62.95 \pm 1.90$ & \\
\hline
\end{tabular}

Table 4. Mean and standard deviation of general fatigue score in the studied groups at the time of study

\begin{tabular}{lccc}
\hline Time & Aromatherapy & Massage therapy & Control \\
\hline Before intervention & $12.61 \pm 3.19$ & $13.03 \pm 1.97$ & $12.71 \pm 2.51$ \\
After 5th session & $12.58 \pm 3.16$ & $12.38 \pm 1.80$ & $12.84 \pm 2.53$ \\
After 10th session & $12.39 \pm 3.24$ & $11.97 \pm 1.49$ & $13.10 \pm 2.48$ \\
Total & $12.53 \pm 0.44$ & $12.46 \pm 0.44$ & $12.88 \pm 0.44$ \\
\hline
\end{tabular}

0.567). At all intervals, the physical fatigue score of the control group was higher than the other two groups. The physical fatigue score in the fifth session was significantly higher when compared to that before intervention, but in the tenth session, it was lower when compared to that before intervention. However, in the massage therapy group, the physical fatigue score decreased at the end of the tenth session (Table 5).

The repeated measures ANOVA results showed that there was a significant difference in the mean score of the reduced activity dimension among the three groups $(P=0.010)$. The mean score was significantly lower in the massage therapy group than in the other two groups $(P<0.001)$. No difference in the score of the reduced activity dimension between the fifth session and the tenth session was observed, but the mean scores at different intervals after intervention were significantly different when compared to those before intervention. The mean scores of the reduced activity dimension at different intervals in different groups were significantly different $(P<0.001)$. The mean score of the reduced activity dimension in the massage therapy group significantly decreased over time compared to the other groups. In the control group, the score in the fifth session decreased when compared to that before intervention and in the tenth session, it increased when compared to that in the fifth session, but the score did not change over time (Table 6).

The repeated measures ANOVA results showed that no statistically significant difference in the scores of the reduced motivation domain was observed among the groups $(P=0.640)$. The mean score of the reduced motivation domain at the studied intervals were significantly different $(P<0.001)$. The scores on the reduced motivation domain at different intervals after intervention were significantly different when compared to those before intervention, but no difference in this domain between the tenth and fifth sessions was observed. There was a significant difference between the mean scores of the reduced motivation dimension among the groups at different intervals $(P<0.001)$. The downward trend in the reduced motivation domain in the massage therapy group remained constant from the beginning of the intervention to the tenth session, but this trend did not significantly change in the other groups (Table 7).

Results from repeated measures ANOVA regarding mental fatigue showed that there was a significant difference in the mean score of mental fatigue between the control group and the aromatherapy group $(P=0.028)$, but not between the control group and the massage therapy group. The mean scores of mental fatigue at different intervals were not significantly different $(P=0.300)$. The mean scores of mental fatigue at different intervals between the groups were significantly different. At different intervals, the mean scores of mental fatigue were higher in the control group when compared to the other two groups (Table 8). The score in the fifth session decreased when compared to before intervention in the aromatherapy group but increased in the tenth session when compared to that in the fifth session. In the massage therapy group, an inverse change in the score of mental fatigue was observed.

\section{Discussion}

The effects of the intervention on the trend of changes in the fatigue score showed that the changes in fatigue in the

Table 5. Mean and standard deviation of physical fatigue score in the studied groups at the time of study

\begin{tabular}{lccc}
\hline Time & Aromatherapy & Massage therapy & Control \\
\hline Before intervention & $12.29 \pm 3.72$ & $12.90 \pm 2.10$ & $13.77 \pm 2.38$ \\
After 5th session & $12.32 \pm 3.74$ & $12.74 \pm 2.03$ & $13.81 \pm 2.48$ \\
After 10th session & $12.26 \pm 3.71$ & $12.52 \pm 1.93$ & $13.68 \pm 2.45$ \\
Total & $12.29 \pm 0.50$ & $12.72 \pm 0.50$ & $13.77 \pm 0.50$ \\
\hline
\end{tabular}


Table 6. Mean and standard deviation of activity reduction score in the studied groups at the time of study

\begin{tabular}{lccc}
\hline Time & Aromatherapy & Massage therapy & Control \\
\hline Before intervention & $12.13 \pm 3.89$ & $10.68 \pm 3.47$ & $12.65 \pm 2.89$ \\
After 5th session & $12.06 \pm 3.99$ & $9.97 \pm 3.22$ & $12.45 \pm 3.16$ \\
After 10th session & $12.06 \pm 3.99$ & $9.58 \pm 2.98$ & $12.61 \pm 2.65$ \\
Total & $12.09 \pm 0.60$ & $10.08 \pm 0.60$ & $11.49 \pm 3.61$ \\
\hline
\end{tabular}

Table 7. Mean and standard deviation of motivation reduction score in the studied groups at the time of study

\begin{tabular}{lccc}
\hline Time & Aromatherapy & Massage therapy & Control \\
\hline Before intervention & $11.00 \pm 2.68$ & $10.87 \pm 2.16$ & $10.87 \pm 2.19$ \\
After 5th session & $10.97 \pm 2.65$ & $10.26 \pm 2.08$ & $10.84 \pm 2.16$ \\
After 10th session & $10.97 \pm 2.60$ & $10.23 \pm 2.03$ & $10.84 \pm 2.10$ \\
Total & $10.98 \pm 0.41$ & $10.45 \pm 0.41$ & $10.85 \pm 0.41$ \\
\hline
\end{tabular}

Table 8. Mean and standard deviation of mental fatigue score in the studied groups at the time of study

\begin{tabular}{lccr}
\hline Time & Aromatherapy & Massage therapy & Control \\
\hline $11.29 \pm 2.51$ & $11.65 \pm 1.74$ & $12.77 \pm 3.10$ & $11.90 \pm 2.56$ \\
$11.13 \pm 2.46$ & $11.87 \pm 1.77$ & $12.94 \pm 3.15$ & $11.98 \pm 2.60$ \\
$11.29 \pm 2.18$ & $11.81 \pm 1.80$ & $12.97 \pm 3.04$ & $12.02 \pm 2.47$ \\
$11.24 \pm 0.44$ & $11.77 \pm 0.44$ & $12.89 \pm 0.44$ & $10.68 \pm 2.57$ \\
\hline
\end{tabular}

massage therapy and control groups were not significant, while in the L. angustifolia aromatherapy group, the changes were significant so that the scores of fatigue reduced after intervention.

One of the reasons that can be mentioned for the lack of a significant effect of massage on the fatigue of people with knee osteoarthritis is the short duration of intervention that was scheduled for 10 sessions.

The results of this study are in contradiction with the results of the study Özdemir et al with the aim of examining the effect of massage on the severity of fatigue and muscle cramps in patients with end-stage renal disease. The results of that study showed that massage had a positive effect on the fatigue in the patients (25). In another study by Sakamoto et al to investigate the psychoneuroimmunologic effects of massage during the postpartum period, the massage was found to decrease fatigue (26).

The results showed that the effect of the aromatherapy on mental fatigue was greater than that of massage therapy. In another study by Lee, on the effect of aromatic oils of lavender and eucalyptus on the postpartum fatigue and quality of sleep, the aromatic oils were observed to significantly affect postpartum fatigue, which is consistent with the results of the present study. The reason for this result is the clinical effects of this essential oil, including suppressing the sympathetic system, improving mood, and reducing stress (27) which is consistent with the study of Sakamato et al to determine the effect of lavender essential oil during rest on the performance of male students (26). The effect of essential oil on the central nervous system is very prominent. The essential oil reduces fatigue by producing physiological and psychological effects $(1,28)$. The fragrance is a stimulant and is warm-natured and penetrating with respect to its smell and effect. Since the aromatherapy facilitates secretion and release of bile, it is used in mental-psychological diseases. The use of this method is useful for general weakness, excessive fatigue, and lethargy as well as during recovery (8).

Reduced motivation, physical fatigue, and general fatigue are other dimensions of fatigue whose scores did not change significantly and no difference in these dimensions among the 3 groups was observed. In the study of van Weert et al, the highest score was attained for the general fatigue dimension and the lowest score for the reduced motivation dimension (29). In the study of Biniyaz et al, comparatively higher mean scores were attained for the physical fatigue and general fatigue dimensions than the other dimensions of fatigue (7).

The effectiveness of the methods studied, such as massage therapy and aromatherapy, requires frequent use of them and comprehensive support from the patients. On the other hand, if patients are not encouraged to use these methods, the psychological and physical effects of these methods in the patient are reduced so that over time, they will feel that the use of these methods does not lead to an appropriate therapeutic effect on their well-being, and therefore they will abandon taking them. More clearly, in people with osteoarthritis, effort should be made to create a positive attitude toward the effects of non-threatening and repeatable methods. Thus, the patients will be able to take advantage of both physiological and emotional effects 
of such methods so that the effects of these methods can be used to improve patients' health (8-10).

\section{Conclusion}

Since osteoarthritis is a disease that has no definite treatment, it is recommended to apply aromatherapy to reduce fatigue in patients with knee osteoarthritis.

\section{Acknowledgments}

This article was derived from a master's thesis on the internal-surgical nursing (ethics code: 1394, 43-IRSKUMS-REC and project No.: 434-1394) at the Research Deputy of Faculty of Nursing and Midwifery, Shahrekord University of Medical Sciences, Shahrekord, Iran. We thank all the patients and the colleagues who collaborated in this work.

\section{Authors' contributions}

FK, AHD and FD conceived the study and contributed reagents and tools. FK and AHD performed the experiments. AHD and FD analyzed the data and drafted the final manuscript. All authors read, revised and approved the final manuscript.

\section{Conflict of interests}

The authors declared no conflict of interest.

\section{Ethical considerations}

This study was approved by the Ethics Committee of Shahrekord University of Medical Sciences (1394,43-IRSKUMS-REC). Also, the study protocol was also registered in the Iranian Registry of Clinical Trials (identifier: IRCT2016062113768N8).

\section{Funding/Support}

This research project was funded by the Research and Technology Deputy of Shahrekord University of Medical Sciences (Grant No. 2104).

\section{References}

1. Shojaedin SS, Sayyah M, Mehrabian H, Sheikh Oveisi J, Barati AH, Razi M. Comparing the radiographic grading scales, knee pain, signs, movement dysfunctions and the quality of life related to osteoarthritis in ex-elite track and field athletes and non-athletes. Feyz Feyz. 2012;16(1):58-64. [Persian].

2. Pollard H, Ward G, Hoskins W, Hardy K. The effect of a manual therapy knee protocol on osteoarthritic knee pain: a randomised controlled trial. J Can Chiropr Assoc. 2008;52(4):229-42.

3. Tahmasebi MN, Motaghi A, Shahrezaee M. Total knee arthroplasty in patients with osteoarthritis: Results of 34 operations. Tehran Univ Med J. 2009;67(2):146-50.

4. Lawrence RC, Helmick CG, Arnett FC, Deyo RA, Felson DT, Giannini EH, et al. Estimates of the prevalence of arthritis and selected musculoskeletal disorders in the
United States. Arthritis Rheum. 1998;41(5):778-99. doi: 10.1002/1529-0131(199805)41:5<778::aid-art4>3.0.co;2-v.

5. Ghodsbin F, Najafi S, Razeghi M, Zare N, Keshtkaran Z. The impact of self care education on quality of life of those clients suffering from osteoarthrities in rehabilation centers of shiraz university of medical science (Iran). J Babol Univ Med Sci. 2010;12(54):65-70.

6. Salehi K, Hamidizadeh S, Mohammadi E ,Torkaman G, Hosaini SM. The effect of Trans-cutaneous Electrical Nerve Stimulation (TENS) on range of motion and morning stiffness of knee joint on patient with knee osteoarthritis. J Shahrekord Univ Med Sci. 2007;9(2):62-7.

7. Biniyaz V, Tayebi A, Sadeghi Shermeh M, Ebadi A, Nemati E. The effect of supplementation with intravenous vitamin $\mathrm{C}$ on fatigue of hemodialysis patients. Journal of Critical Care Nursing. 2013;6(3):145-54.

8. Perlman AI, Sabina A, Williams AL, Njike VY, Katz DL. Massage therapy for osteoarthritis of the knee: a randomized controlled trial. Arch Intern Med. 2006;166(22):2533-8. doi: 10.1001/archinte.166.22.2533.

9. Naderi Z, Mozaffari-Khosravi H, Dehghan A, Fallah Hosseini H, Nadjarzadeh A. The Effect of Ginger (Zingiber Officinale) Powder Supplement on Pain in Patients with Knee Osteoarthritis: a Double-Blind Randomized Clinical Trial. J Shahid Sadoughi Univ Med Sci. 2013;20(5):657-67.

10. Taghizadeh P, Hekmatpou D, Rahzani K, Kazerani H, Rafiei M. Comparing of the effect of reflexive and stroke massages on physiologic indices in patients with MI. Complementary Medicine Journal of Faculty of Nursing \& Midwifery. 2013;2(4):279-90.

11. Hasanpour Dehkordi A. Influence of yoga and aerobics exercise on fatigue, pain and psychosocial status in patients with multiple sclerosis: a randomized trial. J Sports Med Phys Fitness. 2016;56(11):1417-22.

12. Hasanpour-Dehkordi A, Jivad N, Solati K. Effects of Yoga on physiological indices, anxiety and social functioning in multiple sclerosis patients: a randomized trial. J Clin Diagn Res. 2016;10(6):Vc01-vc5. doi: 10.7860/ jcdr/2016/18204.7916.

13. Tahmasebi H, Darvishi A, Zafari M. The effect of inhalation of lavender essential oil on blood pressure, pulse rate and respiratory rate of patients before coronary angiography. Journal of Nursing and Midwifery. 2015;12(11):1002-17.

14. Aourell M, Skoog M, Carleson J. Effects of Swedish massage on blood pressure. Complement Ther Clin Pract. 2005;11(4):242-6. doi: 10.1016/j.ctcp.2005.02.008.

15. Olapour A, Behaeen K, Akhondzadeh R, Soltani F, Al Sadat Razavi F, Bekhradi R. The Effect of Inhalation of Aromatherapy Blend containing Lavender Essential Oil on Cesarean Postoperative Pain. Anesth Pain Med. 2013;3(1):203-7. doi: 10.5812/aapm.9570.

16. Aliasgharpour M, Mohammadi N, Kazemnejad A, Abbaszadeh R. Comparison the effect of lidocaine gel and inhalation of lavender aromatherapy on pain score of arteriovenous fistula puncture in hemodialysis patients. Complementary Medicine Journal of Faculty of Nursing \& Midwifery. 2015;4(4):1000-11.

17. Mirmohamad Aliei M, Khazaie F, Rahnama P, Rahimikian F, Modarres M, Bekhradi R, et al. Effect of Lavender on Pain during Insertion of Intrauterine Device: A Clinical 
Trial. J Babol Univ Med Sci. 2013;15(4):93-9. doi: 10.18869/ acadpub.jbums.15.4.93.

18. Fillion L, Gelinas C, Simard S, Savard J, Gagnon P. Validation evidence for the French Canadian adaptation of the Multidimensional Fatigue Inventory as a measure of cancer-related fatigue. Cancer Nurs. 2003;26(2):143-54.

19. Schneider RA. Reliability and validity of the Multidimensional Fatigue Inventory (MFI-20) and the Rhoten Fatigue Scale among rural cancer outpatients. Cancer Nurs. 1998;21(5):370-3.

20. Shamsi A, Yaghmaei F, Zaeri F. Validity and reliability of "Multidimensional Symptoms Fatigue Inventory-Short Form"(MSFI-SF). J Ilam Univ Med Sci. 2014;22(5):63-9.

21. Lange G, Steffener J, Cook DB, Bly BM, Christodoulou C, Liu WC, et al. Objective evidence of cognitive complaints in Chronic Fatigue Syndrome: a BOLD fMRI study of verbal working memory. Neuroimage. 2005;26(2):513-24. doi: 10.1016/j.neuroimage.2005.02.011.

22. Strauss B, Brix C, Fischer S, Leppert K, Fuller J, Roehrig $\mathrm{B}$, et al. The influence of resilience on fatigue in cancer patients undergoing radiation therapy (RT). J Cancer Res Clin Oncol. 2007;133(8):511-8. doi: 10.1007/s00432-0070195-z.

23. Najafi S, Pashandi S, Mahmoudi H, Ebadi A. The relationship of fatigue with spirometry parameters in veterans with respiratory disorders. Iranian Journal of War and Public Health. 2010;8(2):29-35.

24. Falk K, Swedberg K, Gaston-Johansson F, Ekman I. Fatigue is a prevalent and severe symptom associated with uncertainty and sense of coherence in patients with chronic heart failure. Eur J Cardiovasc Nurs. 2007;6(2):99-104. doi: 10.1016/j.ejcnurse.2006.05.004.

25. Özdemir G, Ovayolu N, Ovayolu O. The effect of reflexology applied on haemodialysis patients with fatigue, pain and cramps. Int J Nurs Pract. 2013;19(3):265-73. doi: 10.1111/ ijn.12066.

26. Sakamoto R, Minoura K, Usui A, Ishizuka Y, Kanba S. Effectiveness of aroma on work efficiency: lavender aroma during recesses prevents deterioration of work performance. Chem Senses. 2005;30(8):683-91. doi: 10.1093/chemse/ bji061.

27. Lee SH. Effects of Aroma Inhalation on Fatigue and Sleep Quality of Postpartum Mothers. Korean J Women Health Nurs. 2004;10(3):235-43.

28. Mangione KK, McCully K, Gloviak A, Lefebvre I, Hofmann M, Craik R. The effects of high-intensity and low-intensity cycle ergometry in older adults with knee osteoarthritis. J Gerontol A Biol Sci Med Sci. 1999;54(4):M184-90.

29. van Weert E, Hoekstra-Weebers J, Otter R, Postema K, Sanderman R, van der Schans C. Cancer-related fatigue: predictors and effects of rehabilitation. Oncologist. 2006;11(2):184-96. doi: 10.1634/theoncologist.11-2-184. 